\title{
On the structure of kinematic systems with complete symmetry
}

\author{
Jochen Trumpf, Robert Mahony, and Tarek Hamel
}

\begin{abstract}
This paper provides a new perspective on the structure of kinematic systems with complete symmetry. These systems naturally occur as models for mechanical systems with symmetry, for example flying or submersible robots. The configuration space of such systems is a homogeneous space of the symmetry Lie group, and it is well known that their kinematics can be lifted to equivariant kinematics on the symmetry group thus allowing global state observer constructions. We provide explicitly checkable sufficient differential-algebraic conditions on the symmetry that will lead to a lifted system in the form of standard left or right invariant kinematics on the symmetry group. Previously known conditions for one of these two cases required finding a velocity lift map with particular properties for which there was no general construction known.
\end{abstract}

\section{INTRODUCTION}

Systems on Lie groups and their homogeneous spaces have been studied extensively since the early 1970 s, starting with the work of [5], [6] and [8]. Brockett's work was motivated by analytical mechanics and the study of mechanical systems, see [7].

Aghannen et al. [1] first recognized the importance of invariance properties of observers for mechanical systems with symmetry. More recent work on understanding the generic structure of observers for invariant systems on Lie groups and homogeneous spaces, [3], [11], [9], has lead to an understanding of the role of invariance properties of observer designs in relation to the resulting observer error dynamics, see [4], [10], [13], [12].

In this paper we close a gap in the structure theory for kinematic systems with complete symmetry [12]. Good global observer constructions only exist in the case where the kinematics lift to standard invariant kinematics on the symmetry Lie group. We provide simple and explicitly checkable sufficient differential-algebraic conditions on the system symmetry for when this is the case and illustrate the new result in two standard examples.

This paper is structured as follows. Section II contains a brief introduction to kinematic systems with complete symmetry. In Section III we show how to embed the system kinematics into a "larger" system with a canonical velocity space. The main results for the two prevalent types of kinematic systems with complete symmetry are presented in Sections III-A and III-B, respectively. Section IV concludes the paper.

This work was partially supported by the Australian Research Council through the ARC Discovery Project DP160100783 "Sensing a complex world: Infinite dimensional observer theory for robots"

Robert Mahony and Jochen Trumpf are with the Research School of Engineering, Australian National University, Canberra, Australia (Jochen. Trumpf|Robert. Mahony) danu.edu.au

Tarek Hamel is with I3S UNS-CNRS, Sophia Antipolis, France thamelei3s.unice.fr

\section{SymmetriC KinematiC SYSTEMS}

Let $M$ be a smooth (finite-dimensional, real) manifold and $T M$ its tangent bundle. Let $V$ be a (finite-dimensional, real) vector space. We study systems of the form

$$
\dot{x}=f(x, v),
$$

where $f: M \times V \rightarrow T M$ is a smooth vector bundle homomorphism, meaning that $f(x, v) \in T_{x} M$ for all $x \in M$ and $v \in V$, and the partial maps $f(x, \cdot): V \rightarrow T_{x} M$ are linear for all $x \in M$. We can think of systems of the form (1) as modelling the kinematics of mechanical systems with configuration state $x \in M$ and kinematic velocity $v$.

Let $G$ be a Lie group and recall that a smooth map

$$
\alpha: G \times M \rightarrow M
$$

is called a right (resp. left) action of $G$ on $M$ if $\alpha(e, x)=$ $x$ and $\alpha(g, \alpha(h, x))=\alpha(h g, x)$ (resp. $\alpha(g, \alpha(h, x))=$ $\alpha(g h, x))$ for all $g, h \in G$ and all $x \in M$. Here, $e \in G$ denotes the identity element of the group. If we do not wish to emphasize the handedness (right or left), we simply use the term action for such a map. Denote the partial maps $\alpha(g, \cdot): M \rightarrow M$ by $\alpha_{g}$ and $\alpha(\cdot, x): G \rightarrow M$ by $\alpha_{x}$, respectively. Note that $\alpha_{g}$ is a diffeomorphism of $M$ for all $g \in G$. Recall that an action $\alpha$ of $G$ on $M$ is called transitive if for every pair $p, q \in M$ there exists $g \in G$ such that $q=\alpha(g, p)$.

Definition 1: [12] Consider a pair of actions

$$
\phi: G \times M \rightarrow M, \quad \psi: G \times V \rightarrow V
$$

of a Lie group $G$ on $M$ and $V$, respectively. The triple $(G, \phi, \psi)$ is called a complete symmetry of system (1) if the action $\phi$ is transitive and

$$
d \phi_{g}(x)[f(x, v)]=f\left(\phi_{g}(x), \psi_{g}(v)\right)
$$

for all $g \in G, x \in M$ and $v \in V$.

We use the term complete right (resp. left) symmetry if we wish to emphasize the handedness of the symmetry actions. Note that transitivity of the action $\phi$ is equivalent to $\phi_{x}$ being surjective for all $x \in M$. The following two examples [12] will serve to illustrate the theory developed in this paper.

Example 1.1: A physical direction of an inertial feature (such as the magnetic field of the earth) relative to a bodyfixed frame (of a robotic vehicle to which a suite of magnetometers is attached) can be modeled as a direction on the two-sphere $\mathrm{S}^{2}$ embedded in $\mathbb{R}^{3}$. As the robotic vehicle rotates the physical direction of the (inertially known) magnetic field moves relative to the body-fixed frame. Such kinematics are important in attitude estimation for mobile robotic vehicles. 
Given the configuration space $M=\mathrm{S}^{2} \subset \mathbb{R}^{3}$, the kinematics considered are

$$
\dot{x}=x \times v,
$$

where $x \in M, v \in V=\mathbb{R}^{3}$ and $\times$ denotes the vector product.

The state $x \in \mathrm{S}^{2}$ is the direction of the inertial feature relative to the body-fixed frame and as an element of the coordinate space $\mathbb{R}^{3}$ is expressed in body-fixed coordinates. Note that the actual state of the vehicle is two-dimensional, while the parametrisation that we use is the embedding into $\mathbb{R}^{3}$, leading to a three-dimensional coordinate representation. The physical velocity $f(x, v)=x \times v$ of the system (an element of $T_{x} \mathrm{~S}^{2}$ ), is the motion of the inertial feature relative to the body-fixed frame. However, this two-dimensional velocity can only be globally parameterised via a threedimensional object $v$. Physically, $v$ is the angular velocity of the body-fixed frame relative to the inertial frame. As an element of the coordinate space $v \in \mathbb{R}^{3}$ it is expressed in body-fixed coordinates.

The special orthogonal group $\mathrm{SO}(3)$ with actions

$$
\phi(Q, x)=Q^{\top} x, \quad \psi(Q, v)=Q^{\top} v,
$$

is a complete right symmetry for this example. The $\mathrm{SO}(3)$ symmetry expresses the physical fact that the laws of motion, in this case just the first order kinematics, do not depend on the orientation of the vehicle.

Example 2.1: A unicycle kinematic system, typically physically realized by two parallel wheels with castors front and back to keep the vehicle from tipping, is one of the most studied non-holonomic systems in the control literature (see, e.g. [2]). The kinematic state of the system can be represented by the position and orientation of the vehicle on a planar surface, the ground plane. Its speed and angular velocity are measured using tachometers on each driving wheel individually. In a typical robotics experiment the vehicle position (but not its orientation) is measured using an overhead camera.

The configuration space considered is $M=\mathbb{R}^{2} \times \mathrm{S}^{1}$. The unicycle kinematics are given by

$$
\begin{aligned}
\dot{\xi}_{1} & =\cos (\theta) u, \\
\dot{\xi}_{2} & =\sin (\theta) u, \\
\dot{\theta} & =q
\end{aligned}
$$

for $x=\left(\left(\xi_{1}, \xi_{2}\right)^{\top}, \theta\right) \in M$ and velocity $v=(u, q) \in V=$ $\mathbb{R}^{2}$.

The state $x=\left(\left(\xi_{1}, \xi_{2}\right)^{\top}, \theta\right) \in \mathbb{R}^{2} \times \mathrm{S}^{1}$ is the position and orientation of the unicycle with respect to an inertial frame, written in inertial coordinates as an element of the coordinate space $\mathbb{R}^{2} \times \mathbb{R}$. The physical velocity $f(x, v)=$ $\left((\cos (\theta) u, \sin (\theta) u)^{\top}, q\right)$ of the system is the motion of the unicycle with respect to the inertial frame, expressed in inertial coordinates. The system is non-holonomic and there is a velocity constraint that enables one to parameterise the physical velocity with two real parameters $u$, the scalar speed (translational velocity), and $q$ the angular velocity, $(u, q) \in V=\mathbb{R}^{2}$.

The special Euclidean group $\mathrm{SE}(2)$ is the set of rigidbody transformations of two-dimensional Euclidean space. An element of $Q \in \mathrm{SE}(2)$ is parameterized by a rotation

$$
R(\alpha)=\left(\begin{array}{cc}
\cos (\alpha) & -\sin (\alpha) \\
\sin (\alpha) & \cos (\alpha)
\end{array}\right)
$$

and a translation $z \in \mathbb{R}^{2}$. The classical homogeneous coordinates of $Q$ are given by

$$
Q=\left(\begin{array}{cc}
R(\alpha) & z \\
0 & 1
\end{array}\right)
$$

The group $\mathrm{SE}(2)$ with actions

$$
\begin{aligned}
\phi(Q,(\xi, \theta)) & =(R(\alpha) \xi+z, \alpha+\theta), \\
\psi(Q, v) & =v,
\end{aligned}
$$

is a complete left symmetry for this example.

It is straightforward to show that $\phi$ is a left action by representing the state $x=(\xi, \theta)$ in homogeneous coordinates as

$$
x^{h}=\left(\begin{array}{cc}
R(\theta) & \xi \\
0 & 1
\end{array}\right)
$$

and noting that $\phi(Q, x)^{h}=Q x^{h}$, i.e. the action $\phi$ corresponds to left matrix multiplication in homogeneous coordinates. This also implies that the action $\phi$ is transitive on $M$. The trivial group action $\psi$ is both right and left handed. The $\mathrm{SE}(2)$ symmetry expresses the physical fact that the kinematics of the unicycle do not depend on its pose.

\section{VELOCITY LIFTS}

In this section we will start to deviate from the development in our previous work [12]. That work was focussed on constructing lifted kinematics for system (1) on the symmetry group $G$ directly. In contrast, here we show how to use a velocity lift to construct an embedding of the trajectories of system (1) into the trajectories of a "larger" kinematic system with complete symmetry on the same configuration space $M$ but with velocity space $\mathfrak{g}$, the Lie algebra of the symmetry group $G$. We give two different sufficient conditions for when this is possible, corresponding to what we termed Type I (resp. Type II) systems in [12].

As a consequence of this different perspective, it becomes clear that Type I (resp. Type II) is in actual fact a property of the symmetry and not of the velocity lift as was previously thought.

In order to construct velocity lifts, we need to fix a reference point $x_{0} \in M$. Given a complete symmetry for system (1), the linear map

$$
\mathrm{d} \phi_{x_{0}}(e): \mathfrak{g} \rightarrow T_{x_{0}} M
$$

is surjective.

and hence has a linear right inverse $r_{x_{0}}: T_{x_{0}} M \rightarrow \mathfrak{g}$ with

$$
\mathrm{d} \phi_{x_{0}}(e) \circ r_{x_{0}}[\xi]=\xi
$$


for all $\xi \in T_{x_{0}} M$. Given a splitting $\mathfrak{g}=\operatorname{ker} \mathrm{d} \phi_{x_{0}}(e) \oplus \mathfrak{h}$, the restriction of $\mathrm{d} \phi_{x_{0}}(e)$ to $\mathfrak{h}$ is a linear bijection and its inverse is a right inverse for $\mathrm{d} \phi_{x_{0}}(e)$. All linear right inverses can be constructed this way by choosing the direct summand $\mathfrak{h}$.

To every right right inverse $r_{x_{0}}$ of $\mathrm{d} \phi_{x_{0}}(e)$ there corresponds a linear map,

$$
\lambda\left(r_{x_{0}}\right): V \rightarrow \mathfrak{g}, v \mapsto r_{x_{0}}\left[f\left(x_{0}, v\right)\right] .
$$

that makes the diagram of linear maps

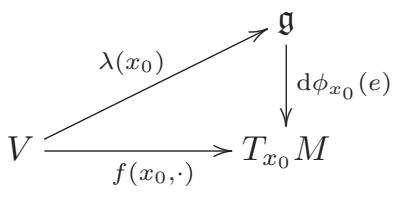

commute. Obviously, the diagram still commutes if we add a linear map $l: V \rightarrow \operatorname{ker} \mathrm{d} \phi_{x_{0}}(e)$ to $\lambda\left(x_{0}\right)$. Conversely, every linear map $\lambda\left(x_{0}\right)$ that makes the diagram (8) commute differs from $\lambda\left(r_{x_{0}}\right)$ by such a linear map $l$. We hence denote

$$
\lambda\left(r_{x_{0}}, l\right): V \rightarrow \mathfrak{g}, v \mapsto r_{x_{0}}\left[f\left(x_{0}, v\right)\right]+l[v] .
$$

Definition 2: A velocity lift for system (1) with respect to a reference point $x_{0} \in M$ is a linear map $\lambda\left(x_{0}\right): V \rightarrow \mathfrak{g}$ that makes the diagram (8) commute.

Example 1.2: Recall the scenario described in Example 1.1. The Lie algebra of $\mathrm{SO}(3)$ is the set of skew symmetric $3 \times 3$ matrices

$$
\mathfrak{s o}(3)=\left\{W \in \mathbb{R}^{3 \times 3} \mid W=-W^{\top}\right\} .
$$

Fix $x_{0}=\mathbf{e}_{3} \in \mathrm{S}^{2}$ where $\mathbf{e}_{3}=(0,0,1)^{\top}$ is the unit vector in the third axis of $\mathbb{R}^{3}$. The velocity lift

$$
\lambda\left(r_{x_{0}}, l\right)[v]=\left(\begin{array}{ccc}
0 & -v_{3} & v_{2} \\
v_{3} & 0 & -v_{1} \\
-v_{2} & v_{1} & 0
\end{array}\right)=: v_{\times} .
$$

corresponds to the right inverse

$$
r_{x_{0}}\left[\left(\xi_{1}, \xi_{2}, 0\right)^{\top}\right]=\left(\begin{array}{ccc}
0 & 0 & -\xi_{2} \\
0 & 0 & \xi_{1} \\
\xi_{2} & -\xi_{1} & 0
\end{array}\right)
$$

and the linear functional

$$
l[v]=\left(\begin{array}{ccc}
0 & -v_{3} & 0 \\
v_{3} & 0 & 0 \\
0 & 0 & 0
\end{array}\right) .
$$

Example 2.2: Recall the scenario described in Example 2.1. The Lie algebra of $\mathrm{SE}(2)$ is the set

$$
\mathfrak{s e}(2)=\left\{\left(\begin{array}{ccc}
0 & a & w_{1} \\
-a & 0 & w_{2} \\
0 & 0 & 0
\end{array}\right) \in \mathbb{R}^{3 \times 3} \mid a, w_{1}, w_{2} \in \mathbb{R}\right\} .
$$

Fix $x_{0}=(0,0,0) \in M$ corresponding to the origin of the inertial frame with zero orientation. The velocity lift

$$
\lambda\left(r_{x_{0}}\right)[(u, q)]=\left(\begin{array}{ccc}
0 & q & u \\
-q & 0 & 0 \\
0 & 0 & 0
\end{array}\right)
$$

corresponds to the right inverse

$$
r_{x_{0}}\left[\left((u, 0)^{\top}, q\right)\right]=\left(\begin{array}{ccc}
0 & q & u \\
-q & 0 & 0 \\
0 & 0 & 0
\end{array}\right) .
$$

\section{A. Type II symmetries}

In our previous work [12], Type II systems were characterized by a trivial velocity action. We turn this into a formal definition.

Definition 3: A complete symmetry $(G, \phi, \psi)$ for system (1) is called (of) Type II if $\psi(g, v)=v$ for all $g \in G$ and all $v \in V$.

Thinking of $\mathrm{d} \phi_{x_{0}}(e)$ as the lifted equivalent of $f\left(x_{0}, \cdot\right)$ and using the defining property (2) of equivariance for $x=$ $\phi_{g}\left(x_{0}\right)$ then motivates the study of the following system map $\bar{f}: M \times \mathfrak{g} \rightarrow T M$,

$$
\bar{f}\left(\phi_{g}\left(x_{0}\right), U\right)=\mathrm{d} \phi_{g}\left(x_{0}\right) \mathrm{d} \phi_{x_{0}}(e)[U] .
$$

Let $\operatorname{stab}\left(x_{0}\right)=\left\{s \in G \mid \phi\left(s, x_{0}\right)=x_{0}\right\} \subset G$ denote the stabilizer subgroup of $x_{0}$ under $\phi$.

Proposition 4: The map $\bar{f}$ in (11) is well defined if $f\left(x_{0}, \cdot\right): V \rightarrow T_{x_{0}} M$ is surjective and $\psi(s, v)=v$ for all $s \in \operatorname{stab}\left(x_{0}\right)$ and all $v \in V$. In this case, $\left(G, \phi, \Psi_{0}\right)$ is a complete symmetry for the system

$$
\dot{x}=\bar{f}(x, U) .
$$

Here, $\Psi_{0}(g, U)=U$ denotes the trivial action of $G$ on $\mathfrak{g}$.

Proof: We show the proof for the case of right handed actions, the proof for the left handed case is entirely analogous. Let $U \in \mathfrak{g}$ then $\mathrm{d} \phi_{x_{0}}(e)[U]=f\left(x_{0}, v\right)$ for some $v \in V$ as $f\left(x_{0}, \cdot\right)$ is surjective. Let $s \in \operatorname{stab}\left(x_{0}\right)$ then

$$
\begin{aligned}
\bar{f}\left(\phi_{s g}\left(x_{0}\right), U\right) & =\mathrm{d} \phi_{s g}\left(x_{0}\right)\left[f\left(x_{0}, v\right)\right] \\
& =f\left(\phi_{s g}\left(x_{0}\right), \psi_{s g}(v)\right) \\
& =f\left(\phi_{g}\left(\phi_{s}\left(x_{0}\right)\right), \psi_{g}\left(\psi_{s}(v)\right)\right) \\
& =f\left(\phi_{g}\left(x_{0}\right), \psi_{g}(v)\right) \\
& =\mathrm{d} \phi_{g}\left(x_{0}\right)\left[f\left(x_{0}, v\right)\right] \\
& =\bar{f}\left(\phi_{g}\left(x_{0}\right), U\right)
\end{aligned}
$$

for all $g \in G$, where the second and fifth equalities follow from the symmetry condition (2). This shows that $\bar{f}$ is well defined.

Now compute

$$
\begin{aligned}
& \mathrm{d} \phi_{h}\left(\phi_{g}\left(x_{0}\right)\right) \bar{f}\left(\phi_{g}\left(x_{0}\right), U\right)= \\
& \mathrm{d} \phi_{h}\left(\phi_{g}\left(x_{0}\right)\right) \mathrm{d} \phi_{g}\left(x_{0}\right) \mathrm{d} \phi_{x_{0}}(e)[U]= \\
& \mathrm{d} \phi_{g h}\left(x_{0}\right) \mathrm{d} \phi_{x_{0}}(e)[U]= \\
& \bar{f}\left(\phi_{g h}\left(x_{0}\right), U\right)= \\
& \bar{f}\left(\phi_{h}\left(\phi_{g}\left(x_{0}\right)\right), U\right)
\end{aligned}
$$

for $g, h \in G$ and $U \in \mathfrak{g}$. This completes the proof.

The following theorem is the main result of this section for Type II symmetries.

Theorem 5: Consider a Type II complete symmetry $(G, \phi, \psi)$ for system (1), fix a reference point $x_{0} \in M$ and 
let $\lambda\left(x_{0}\right): V \rightarrow \mathfrak{g}$ be a velocity lift with respect to $x_{0}$. If $f\left(x_{0}, \cdot\right): V \rightarrow T_{x_{0}} M$ is surjective then the map $(x, v) \mapsto$ $(x, \lambda(v))$ maps trajectories of system (1) to trajectories of system (12).

Proof: This follows immediately from Proposition 8 and

$$
\begin{aligned}
f\left(\phi_{g}\left(x_{0}\right), v\right) & =f\left(\phi_{g}\left(x_{0}\right), \psi_{g}(v)\right) \\
& =d \phi_{g}\left(x_{0}\right)\left[f\left(x_{0}, v\right)\right] \\
& =d \phi_{g}\left(x_{0}\right) \mathrm{d} \phi_{x_{0}}(e) \lambda[v] \\
& =\bar{f}\left(\phi_{g}\left(x_{0}\right), \lambda[v]\right)
\end{aligned}
$$

for all $g \in G$ and $v \in V$.

Remark 6: It is straightforward to show that in the situation of Theorem 5 and in the case of a right handed symmetry, the projection $\phi_{x_{0}}: G \rightarrow M$ maps trajectories of the standard right invariant kinematics $\dot{g}=\mathrm{d} R_{g}[u]$ on $G$ to trajectories of system (12). Here, $R_{g}: G \rightarrow G, h \mapsto h g$ denotes right translation by $g \in G$.

Example 2.3: Example 2.1 is obviously of Type II and the lifted kinematics correspond simply to rewriting the system kinematics in homogeneous coordinates, thereby justifying the use of the term "homogeneous" in this context.

\section{B. Type I symmetries}

The story for Type I systems is at first glance much more complicated [12], however, a careful analysis of what does not work with a lifted system defined by (11) in this case leads to the following formal definition. We only treat the case of right handed symmetries as that matches our Example 1.1 .

Definition 7: A complete right symmetry $(G, \phi, \psi)$ for system (1) is called (of) Type $I$ with respect to a reference point $x_{0} \in M$ if

$$
\operatorname{Ad}_{s} \lambda\left(x_{0}\right)\left[\psi_{s}(v)\right]=\lambda\left(x_{0}\right)[v]
$$

for all $s \in \operatorname{stab}\left(x_{0}\right)$ and $v \in V$ and some velocity lift $\lambda\left(x_{0}\right): V \rightarrow \mathfrak{g}$ with respect to $x_{0}$. Here, $\operatorname{Ad}_{g}$ denotes the adjoint action of $g \in G$ on $\mathfrak{g}$.

From similar considerations as in the Type II case, we are led to study the following system map $\bar{f}: M \times \mathfrak{g} \rightarrow T M$,

$$
\bar{f}\left(\phi_{g}\left(x_{0}\right), U\right)=\mathrm{d} \phi_{\phi_{g}\left(x_{0}\right)}(e)[U] .
$$

In contrast to the Type II scenario, this map is always well defined.

Proposition 8: $\left(G, \phi, \mathrm{Ad}^{-1}\right)$ is a complete symmetry for the system

$$
\dot{x}=\bar{f}(x, U),
$$

where $\bar{f}$ is defined by (14).

Proof: We need to show that $\mathrm{d} \phi_{h}(x) \bar{f}(x, U)=$ $\bar{f}\left(\phi_{h}(x), \operatorname{Ad}_{h^{-1}} U\right)$ for all $h \in G, x \in M$ and $U \in \mathfrak{g}$.
To this end we compute

$$
\begin{aligned}
& \mathrm{d} \phi_{h}\left(\phi_{g}\left(x_{0}\right)\right) \bar{f}\left(\phi_{g}\left(x_{0}\right), U\right)= \\
& \mathrm{d} \phi_{h}\left(\phi_{g}\left(x_{0}\right)\right) \mathrm{d} \phi_{\phi_{g}\left(x_{0}\right)}(e)[U]= \\
& \mathrm{D}_{k} \phi\left(h,\left.\phi\left(k, \phi_{g}\left(x_{0}\right)\right)\right|_{k=e}[U]=\right. \\
& \mathrm{D}_{k} \phi\left(h^{-1} k h,\left.\phi\left(h, \phi_{g}\left(x_{0}\right)\right)\right|_{k=e}[U]=\right. \\
& \mathrm{d} \phi_{\phi\left(h, \phi_{g}\left(x_{0}\right)\right)}(e)\left[\operatorname{Ad}_{h^{-1}} U\right]= \\
& \bar{f}\left(\phi_{h}\left(\phi_{g}\left(x_{0}\right)\right), \operatorname{Ad}_{h^{-1}} U\right) .
\end{aligned}
$$

This completes the proof.

The following theorem is the main result of this section for Type I symmetries.

Theorem 9: Consider a Type I complete right symmetry $(G, \phi, \psi)$ for system (1), fix a reference point $x_{0} \in M$ and let $\lambda\left(x_{0}\right): V \rightarrow \mathfrak{g}$ be a velocity lift with respect to $x_{0}$. Then the map $\lambda: M \times V \rightarrow \mathfrak{g}$ with

$$
\lambda\left(\phi_{g}\left(x_{0}\right), v\right)=\operatorname{Ad}_{g^{-1}} \lambda\left(x_{0}\right)\left[\psi_{g^{-1}}(v)\right]
$$

is well defined and the map $(x, v) \mapsto(x, \lambda(x, v))$ maps trajectories of system (1) to trajectories of system (15).

Proof: That the map $\lambda$ in (16) is well defined follows immediately from condition (13). To complete the proof we compute

$$
\begin{aligned}
f\left(\phi_{g}\left(x_{0}\right), v\right) & =\mathrm{d} \phi_{g}\left(x_{0}\right)\left[f\left(x_{0}, \psi_{g^{-1}}(v)\right]\right. \\
& =\mathrm{d} \phi_{g}\left(x_{0}\right) \mathrm{d} \phi_{x_{0}}(e) \lambda\left(x_{0}\right)\left[\psi_{g^{-1}}(v)\right] \\
& =\mathrm{d} \phi_{\phi_{g}\left(x_{0}\right)}(e) \operatorname{Ad}_{g^{-1}} \lambda\left(x_{0}\right)\left[\psi_{g^{-1}}(v)\right] \\
& =\bar{f}\left(\phi_{g}\left(x_{0}\right), \operatorname{Ad}_{g^{-1}} \lambda\left(x_{0}\right)\left[\psi_{g^{-1}}(v)\right]\right) \\
& =\bar{f}\left(\phi_{g}\left(x_{0}\right), \lambda\left(\phi_{g}\left(x_{0}\right), v\right)\right),
\end{aligned}
$$

for all $g \in G$ and $v \in V$, where we have used the defining property of the velocity lift $\lambda\left(x_{0}\right)$ and the fact that $\mathrm{d} \phi_{g}\left(x_{0}\right) \mathrm{d} \phi_{x_{0}}(e)[U]=\mathrm{d} \phi_{\phi_{g}\left(x_{0}\right)}(e)\left[\operatorname{Ad}_{g^{-1}} U\right]$ for all $g \in G$ and $U \in \mathfrak{g}$.

Remark 10: It is straightforward to show that in the situation of Theorem 9, the projection $\phi_{x_{0}}: G \rightarrow M$ maps trajectories of the standard left invariant kinematics $\dot{g}=$ $\mathrm{d} L_{g}[u]$ on $G$ to trajectories of system (12). Here, $L_{g}: G \rightarrow$ $G, h \mapsto g h$ denotes left translation by $g \in G$.

Example 1.3: It can easily be checked that Example 1.1 is of Type I with respect to any reference point and any associated velocity lift. The system (15) is given by $\bar{f}\left(x, v_{\times}\right)=$ $v_{\times}^{\top} x$ and the lifted kinematics are the standard left invariant rigid body kinematics.

\section{CONCLUSIONS}

We have provided explicitly checkable sufficient differential-algebraic conditions on the complete symmetry of a kinematic system that lead to lifted kinematics of standard invariant type on the symmetry group. This closes a gap in the literature on observer design for kinematic systems with symmetry. The new characterization of Type I symmetries raises interesting questions about the nature of the reference point dependence of the lifted kinematics. This is an interesting topic for future work that will likely require the careful study of adjoint orbits in the symmetry Lie algebra, a classical topic in Lie theory. 


\section{REFERENCES}

[1] N. Aghannan and P. Rouchon. An intrinsic observer for a class of Lagrangian systems. IEEE Transactions on Automatic Control, 48(6):936-945, 2003.

[2] A. M. Bloch. Nonholonomic mechanics and control. Springer, 2003.

[3] S. Bonnabel, P. Martin, and P. Rouchon. Symmetry-preserving observers. IEEE Transactions on Automatic Control, 53(11):2514 2526, 2008.

[4] S. Bonnabel, P. Martin, and P. Rouchon. Non-linear symmetrypreserving observers on Lie groups. IEEE Transactions on Automatic Control, 54(7):1709-1713, 2009.

[5] R. W. Brockett. System theory on group manifolds and coset spaces. SIAM Journal on Control, 10(2):265-284, 1972.

[6] R. W. Brockett. Lie theory and control systems defined on spheres. SIAM Journal on Applied Mathematics, 25(2):213-225, 1973.

[7] R. W. Brockett. Control theory and analytical mechanics. In C. Martin and R. Hermann, editors, Geometric Control Theory, volume VII of Lie Groups: History, Frontiers and Applications, pages 1-48. Math. Sci. Press, 1977.

[8] V. Jurdjevic and H. J. Sussmann. Control systems on Lie groups. Journal of Differential Equations, 12:313-329, 1972.

[9] C. Lageman, J. Trumpf, and R. Mahony. Observers for systems with invariant outputs. In Proceedings of the European Control Conference, pages 4587-4592, 2009.

[10] C. Lageman, J. Trumpf, and R. Mahony. Gradient-like observers for invariant dynamics on a Lie group. IEEE Transactions on Automatic Control, 55(2):367-377, 2010.

[11] R. Mahony, T. Hamel, and J.-M. Pflimlin. Non-linear complementary filters on the special orthogonal group. IEEE Transactions on Automatic Control, 53(5):1203-1218, 2008

[12] R. Mahony, J. Trumpf, and T. Hamel. Observers for kinematic systems with symmetry. In Proceedings of the 9th IFAC Symposium on Nonlinear Control Systems (NOLCOS), pages 617-633, 2013.

[13] J. Trumpf, R. Mahony, T. Hamel, and C. Lageman. Analysis of non-linear attitude observers for time-varying reference measurements. IEEE Transactions on Automatic Control, 57:2789-2800, 2012 